\title{
Polypharmacy Among the Elderly in a List-Patient System
}

\author{
Inger Cathrine Kann ${ }^{1}$ Christofer Lundqvist ${ }^{1,2,3} \cdot$ Hilde Lurås ${ }^{1,2}$
}

Published online: 20 August 2015

(C) The Author(s) 2015. This article is published with open access at Springerlink.com

\begin{abstract}
Background Polypharmacy, i.e. the simultaneous use of multiple drugs, is known to be associated with compliance errors and adverse drug reactions. Norway has a list-patient system in general practice, formalizing the relationship between the patient and his/her regular general practitioner (GP). One important aim with a list-patient system is to secure medical quality in primary care by giving the GP the responsibility for coordinating the medical treatment.

Objective To examine the regular GP's role in polypharmacy to the home-dwelling elderly in Norway and to determine by how much multiple prescribers increase the risk of polypharmacy.

Methods This was a population registry study based on data on all prescription drugs dispensed at pharmacies to patients 70 years and older from the Norwegian Prescription Database, merged with data on GPs and GPs' patient lists from the Regular General Practitioner Database. The dataset included 624,308 patients and 4520 GPs in the period from 2004 to 2007. Outcome measures were: number of drug-substances prescribed and dispensed per
\end{abstract}

Christofer Lundqvist

a.c.lundqvist@medisin.uio.no

Inger Cathrine Kann

inger.cathrine.kann@ahus.no

Hilde Lurås

hilde.luras@ahus.no

$1 \quad$ Health Services Research Centre (HØKH), Akershus University Hospital, PO Box 1000, 1478 Lørenskog, Norway

2 Institute of Clinical Medicine, Campus Ahus, University of Oslo, Oslo, Norway

3 Department of Neurology, Akershus University Hospital, Lørenskog, Norway patient by the regular GP, other GPs, non-GP specialists and hospital doctors; polypharmacy, defined as five or more prescribed and dispensed substances in the same quarter; excessive polypharmacy, defined as ten or more prescribed and dispensed substances in the same quarter. Results Polypharmacy is high and increasing despite the list-patient system. GPs prescribe all the substances that cause polypharmacy in $64 \%$ of the incidents, but the patients' risk of polypharmacy increases substantially with number of prescribers, odds ratio 2.32 (95\% CI 2.31-2.33). Conclusion GPs have a major role in the high and increasing polypharmacy among the elderly in Norway. Any intervention intending to improve the situation must necessarily include the GPs.

\section{Key Points}

Prescriptions from the patient's regular GP contribute to the bulk of polypharmacy among the elderly.

Despite a list-patient system in general practice, polypharmacy increases over time.

Patients' risk of polypharmacy increases with the number of prescribers.

\section{Introduction}

Polypharmacy, i.e. the simultaneous use of multiple drugs, is known to be associated with compliance errors and adverse drug reactions [1]. Since multi-morbidity is common among the elderly [2], the involvement of medical 
specialists from different fields is often required [3, 4]. Both the increasing morbidity and the change in the human body with age make adverse drug reactions more likely among the elderly [3-6]. The risk of drug interactions and the probability of compliance errors increases with the quantity of drugs and substances used [7-9]. The number of drug substances is shown to be the most important clinical predictor of adverse drug reactions [10]. A study from Norway indicated that one-third of the elderly Norwegian population is exposed to potentially inappropriate medications [11]. The number of prescribing physicians may be an independent risk factor for adverse drug reactions [12], and studies also reveal a large variation in physicians' individual prescription practice, not least in terms of polypharmacy [13].

Norway has a list-patient system in general practice formalizing the relationship between the patient and his/her regular general practitioner (GP). One important aim with a listpatient system is to secure medical quality in primary care by giving the GP the responsibility for coordinating the medical treatment [14] which gives the GP a unique 'spider-in-theweb' position. The patient may, however, use another GP with whom he/she is not registered during holidays or when no appointments are available for other reasons. The GPs are gatekeepers to specialist health care. Hence, patients generally need a referral from their GP to see a medical specialist.

The aim of this study is to explore the role of the patient's regular GP in a list patient system, with a special reference to polypharmacy. The main issues addressed are: (a) what proportion of multiple drugs and drug substances are prescribed by the patient's regular GP? and (b) does the number of prescribers for the same patient predict polypharmacy?

\section{Methods}

\subsection{Setting and Participants}

Data from the Norwegian Prescription Database (NorPD) of all prescription drugs dispensed at pharmacies to the home-dwelling elderly ( $\geq 70$ years old) were merged with data on GPs and their patient lists from the Regular General Practitioner Database (RPD).

\subsection{Collected Data}

Data from the NorPD are based on electronic registration of every prescription collected at pharmacies in Norway. NorPD is a state-run registry and all pharmacies are compelled to register all their prescriptions as they are expedited. We were given access to the total registry covering the years 2004-2007. Patients and prescribers are given a unique code in the registry which allows prescriptions by the same prescriber as well as prescriptions to one single patient to be followed over time.

For each of the included patients, the medication prescribed by the regular GP, by other GPs and by non-GP specialists and hospital doctors were calculated separately. The variables of interest were aggregated over quarters.

Other GPs are identified as GPs that are found in the RPD database at the time the drugs were dispensed, but are not the patient's regular GP. Non-GP specialists and hospital doctors are identified by not being in the RPD database at the time of dispensing the medication, but registered as a physician in the NorPD database. The methods have previously been described in more detail [15].

\subsection{Outcomes}

Outcomes were: the number of different drug-substances prescribed and dispensed to a patient in the same quarter; the presence of polypharmacy, defined as five or more drug substances prescribed and dispensed to a patient in the same quarter; and presence of excessive polypharmacy, defined as ten or more drug substances prescribed and dispensed to a patient in the same quarter.

The use of five or more substances is a standard cut-off for clinically relevant polypharmacy, while the concurrent use of ten or more substances has been suggested to represent excessive polypharmacy [13].

The classification of drug-substances is based on the Anatomical Therapeutic Chemical (ATC) classification system, in which a drug-substance is defined at the highest level, using all elements in the classification system from WHO [16].

\subsection{Statistics and Analytical Procedures}

Since our dataset covers the whole population of the elderly (70 years and older), descriptive statistics are presented as actually observed values and means without confidence intervals (CIs). In regression analysis, $95 \%$ CIs are presented.

A multiple logistic regression was performed to calculate the association between number of prescribers and polypharmacy, confounders were age and gender. All analyses were conducted in STATA version 10 (Stata Corp LP, College Station, TX, USA).

\section{Results}

\subsection{Population}

The dataset consisted of 624,308 patients, listed with 4520 GPs over 4 years, giving 7,958,068 quarterly patient 
observations. A total of 41 million prescriptions were registered over the observation period.

\subsection{The GPs' Share of Prescribed Drug Substances}

The regular GP prescribed $69 \%$ of the drug-substances, while $9 \%$ were prescribed by other GPs, and $22 \%$ by nonGP specialists and hospital doctors.

The number of drugs per patient is on average 3.6 per quarter (Table 1).

\subsection{The Development of Polypharmacy}

In 2007, the fraction of elderly patients that received at least five drug substances quarterly (polypharmacy) was $36 \%$ (176,626 of 497,538 individuals), while $7 \%(32,340$ of 497,538 individuals) received at least ten drug substances quarterly (excessive polypharmacy).

Further, in 2007 the GP was responsible for more than $64 \%(113,439$ of 176,626$)$ of polypharmacy incidents and $40 \%(12,936$ of 32,340$)$ of excessive polypharmacy incidents (Table 2). The GPs' proportion has increased steadily from 2004 (Fig. 1).

Table 3 shows the results of logistic regression analyses estimating the risk of polypharmacy and excessive polypharmacy with increasing number of prescribers. Number of prescribers was strongly predictive of polypharmacy and excessive polypharmacy (OR 2.32). A positive time trend as well as seasonal variation was observed in both regressions (Fig. 1). The seasonal dummies show that the risk of polypharmacy is lowest in the first quarter, and $40 \%$ higher in the fourth quarter. Patient age and gender were also significant predictors.

\section{Discussion}

\subsection{Summary of Main Findings}

We have shown that the home-dwelling elderly in Norway received a high and increasing number of drug substances between 2004 and 2007. Over this period, polypharmacy and excessive polypharmacy was observed among a mean of 33 and $6 \%$ of the patients, respectively. It increased steadily over time. The GP was responsible for $63 \%$ of the polypharmacy incidents and $39 \%$ of the excessive polypharmacy incidents. Since the GPs prescribe most of the drugs, polypharmacy caused by the regular GP is frequent. The number of prescribing physicians per patient increased the patients' risk of polypharmacy; increasing the number of prescribers by one more than doubled the risk of polypharmacy.

\subsection{Strengths and Limitations}

The data explored in this study are extracted from a registry of all prescriptions dispensed to the total populations of the home-dwelling elderly in Norway as opposed to prescriptions prescribed. Since compliance with prescriptions is a well-known problem [8], this is a considerable strength.
Table 1 Average quarterly number of drug substances per patient, by physician group $(N=7,958,068)$

\begin{tabular}{llllll}
\hline & 2004 & 2005 & 2006 & 2007 & Overall mean \\
\hline Number of drug substances & & & & & \\
From the regular GP & 2.3 & 2.4 & 2.5 & 2.6 & 2.5 \\
From another GP & 0.3 & 0.3 & 0.3 & 0.3 & 0.3 \\
From non-GP specialists and hospital doctors & 0.7 & 0.8 & 0.8 & 0.8 & 0.8 \\
Total & 3.4 & 3.5 & 3.6 & 3.8 & 3.6 \\
\hline
\end{tabular}

$G P$ general practitioner

Table 2 Development in the number of patients experiencing polypharmacy and major polypharmacy

\begin{tabular}{lrrrrrr}
\hline & \multicolumn{1}{c}{2004} & \multicolumn{1}{c}{2005} & 2006 & 2007 & Overall mean & $\%$ change 2004-2007 \\
\hline Polypharmacy & 152,778 & 161,851 & 168,774 & 176,626 & 164,632 & 16 \\
No. of these prescribed by the GP & 95,548 & 102,274 & 107,039 & 113,439 & 104,450 & 19 \\
Excessive polypharmacy & 24,882 & 27,306 & 29,374 & 32,340 & 28,351 & 30 \\
No. of these prescribed by the GP & 8958 & 10,426 & 11,451 & 12,936 & 10,942 & 44 \\
$N$ & 497,648 & 496,475 & 497,857 & 497,538 & 497,379 & 0 \\
Polypharmacy from the GP (\%) & 63 & 63 & 63 & 64 & 63 & 3 \\
Excessive polypharmacy from the GP (\%) & 36 & 38 & 39 & 40 & 39 & 11 \\
\hline
\end{tabular}

$G P$ general practitioner 
Fig. 1 Time trends of the presence of polypharmacy and excessive polypharmacy as well as polypharmacy incidents and excessive polypharmacy incidents prescribed by the general practitioner (GP) alone over the observation period. Prescription in 2004 was set as the starting point within each category
Table 3 Logistic regression. Number of prescribers, season prescribed, and age and gender influences on patients' odds for polypharmacy and excessive polypharmacy (odds ratios for physicians and time are age- and gender-adjusted)

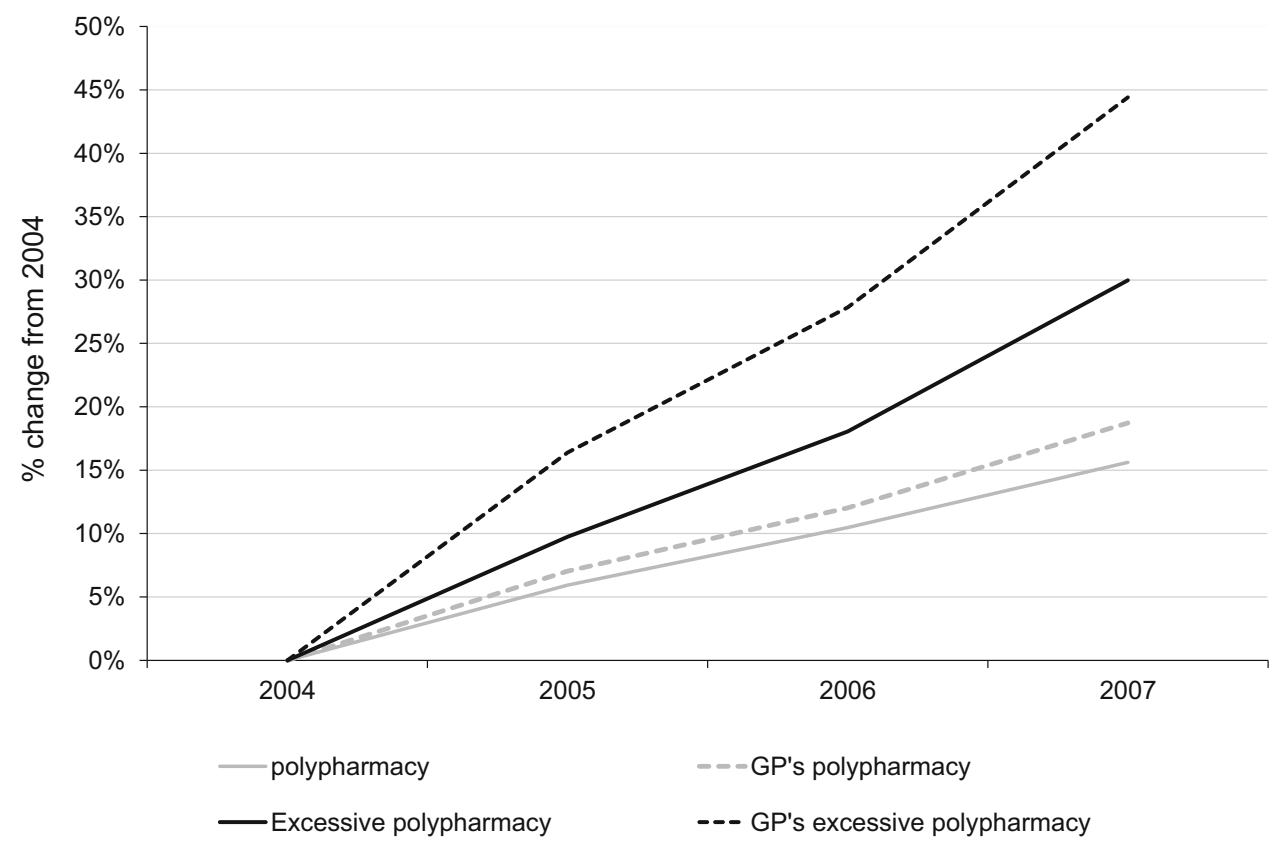

\begin{tabular}{|c|c|c|c|c|c|c|}
\hline & \multicolumn{3}{|c|}{ Polypharmacy } & \multicolumn{3}{|c|}{ Excessive polypharmacy } \\
\hline & Odds ratio & $P$ value & $95 \% \mathrm{CI}$ & Odds ratio & $P$ value & $95 \% \mathrm{CI}$ \\
\hline Number of physicians & 2.323 & $<0.001$ & $2.316-2.331$ & 1.987 & $<0.001$ & $1.978-1.997$ \\
\hline Time trend & 1.078 & $<0.001$ & $1.076-1.080$ & 1.122 & $<0.001$ & $1.118-1.127$ \\
\hline \multicolumn{7}{|c|}{ Season prescribed with first quarter as reference } \\
\hline Quarter 2 & 1.176 & $<0.001$ & $1.171-1.181$ & 1.153 & $<0.001$ & $1.142-1.164$ \\
\hline Quarter 3 & 1.110 & $<0.001$ & $1.105-1.115$ & 1.083 & $<0.001$ & $1.072-1.093$ \\
\hline Quarter 4 & 1.405 & $<0.001$ & $1.399-1.412$ & 1.433 & $<0.001$ & $1.419-1.446$ \\
\hline Patient age & 1.024 & $<0.001$ & $1.023-1.025$ & 1.025 & $<0.001$ & $1.023-1.026$ \\
\hline Patient gender & 1.135 & $<0.001$ & $1.123-1.147$ & 1.158 & $<0.001$ & $1.138-1.178$ \\
\hline
\end{tabular}

Two phenomena may contribute to underestimation of polypharmacy in our dataset. First, GPs in Norway are not obliged to report to the health authorities when patients move into an institution and receive drugs directly from the institution, and, second, the patients' use of over-thecounter drugs is not registered in the database. Thus our data may underestimate the actual drug use, which serves to strengthen the significance of our results as an even higher medication use may be expected if this non-registered data could have been included. However, the estimated size of the population moving into an institution, based on official statistics, suggests this at most to represent $8 \%$ of the elderly.

Our indicator of polypharmacy is drugs purchased within a 3-month period. Bjerrum et al. [9] conclude that from a conceptual point of view, an estimator based on the number of simultaneously used drugs (calculated from the date of purchase) is preferable, but also that the number of drugs purchased in a 3-month period may be a useful estimator. The advantage with this simple and robust measure is that it can be used to compare seasonal differences in the purchasing of drugs.

Factors related to the patient's health and diagnoses, and factors related to the accessibility of high-quality healthcare provision may explain part of the increase in polypharmacy over time. We cannot address these issues through a prescription registry with no medical information other than the prescription itself.

\subsection{Discussion of the Results}

On average patients received 3.6 drugs quarterly. In a study from Denmark of 75-year-olds living in their own homes, the average number of medications per patient was 4.4, which is similar to, albeit slightly higher than, our figures [3]. 
The average number of medicines prescribed for people aged 60 years and over in England has almost doubled per person per year over the past decade [17]. This represents a yearly increase of nearly $7 \%$. In comparison the yearly increase of average amounts per patient in our data is $4 \%$.

A high level and a trend towards increasing polypharmacy among the elderly which we have documented here may also reflect an increasing complexity of highly specialised medical treatment. However, the number of prescribed drugs has also been shown to be one of the most important predictors of adverse drug reactions $[1,10]$, and the elderly are more sensitive to side effects as well as having less metabolic capacity for eliminating excess drugs [3-6]. We therefore suggest that the results described here are worrying and deserve further focus. The finding that the segment with the highest number of drugs (excessive polypharmacy) increases most rapidly supports this.

The risk of polypharmacy increased with the number of prescribers. Thus, a special awareness among GPs toward their patients is needed when many physicians are involved, in order to prevent dangerous combinations. This is of special importance in the presence of complicated illness and multiple co-morbid conditions where more prescribers are likely to be involved. The importance of adequate communication between different physicians is thus also highlighted.

The seasonal difference in polypharmacy is worth noting. The risk is lowest in the first quarter and highest in the fourth. In Norway patients are reimbursed for the cost of drugs when accumulated costs exceed a certain threshold during one calendar year. Thus, in the last quarter of the year many elderly people may stock up drugs because they are more likely to have reached the reimbursement threshold. Knowing that compliance errors are common in the elderly population [3], this may represent a health risk and is thus an example of the direct influence of health economic policies regarding medication reimbursement on prescription-related risk.

Though less frequent, polypharmacy also occurs in the group of patients who received medications only from their regular GP. This finding suggests that the polypharmacy problem needs to be attended to both by measures addressed towards the quality of drug prescriptions by the regular GPs, and by measures improving communication and coordination between different prescribers. We have previously showed that there is a relationship between the number of prescribers and the prescription of addictive medication [15]; polypharmacy may further complete this pattern.

The relationship between the number of prescribers and the number of drugs prescribed is important and justifies the idea of having one physician, e.g. the GP, as the 'controlling agent', with the task of being observant of the risk of, and trying to reduce, such polypharmacy and its negative consequences. However, as the regular GP him-/ herself also contributes importantly to polypharmacy, an increased general awareness of the problem and its consequences among GPs as well as a focus on the practice situation and logistics which may influence prescribing is required. By necessity this requires GPs well trained in clinical pharmacology among the elderly as well as systems supporting GPs in their handling of ever-more specialised medical treatment prescribed by various other specialists.

\section{Conclusion}

The results indicate that the Norwegian list-patient system does not prevent polypharmacy, nor does it prevent an increase in polypharmacy over time. More research is needed to explore the main reasons for the demonstrated frequent and increasing polypharmacy. However, it is clear that no change in prescription practices regarding polypharmacy can be undertaken without involvement of GPs.

Acknowledgments The Norwegian Prescription Database (NorPD), the Norwegian Social Science Data (NSD), the Norwegian Labour and Welfare Administration (NAV) and Statistics Norway (SSB) are gratefully acknowledged for providing data from the Regular Practitioner Database (RPD), making the analyses possible. None of these sources are responsible for the data analyses or the interpretations.

\section{Compliance with Ethical Standards}

Funding The Norwegian Research Council financed this work though the Research Programme on Health and Care Services. We are sincerely grateful for this financial support. Support to CL from the South-East Norway regional health authority is also gratefully acknowledged.

Conflict of interest CL has received research funding from Abbvie Pharmaceuticals for Parkinson research not related to the present work. CL, ICK and HL all affirm that they have no conflicts of interest.

Ethical approval The study was reviewed by and received all necessary approvals from The Norwegian Directorate for Health and Social Affairs and The Norwegian Data Inspectorate. The data are anonymous registry data, and cannot be traced to human subjects. ${ }^{1}$

Open Access This article is distributed under the terms of the Creative Commons Attribution-NonCommercial 4.0 International License (http://creativecommons.org/licenses/by-nc/4.0/), which permits any noncommercial use, distribution, and reproduction in any medium, provided you give appropriate credit to the original author(s) and the source, provide a link to the Creative Commons license, and indicate if changes were made.

\footnotetext{
${ }^{1}$ Since this is a meta-study using non-personally identifiable data, the approvals are sufficient according to Norwegian regulations.
} 


\section{References}

1. Hajjar ER, Cafiero AC, Hanlon JT. Polypharmacy in elderly patients. Am J Geriatr Pharmacother. 2007;5(4):345-51.

2. Marengoni A, Angleman S, Melis R, Mangialasche F, Karp A, Garmen A, et al. Aging with multimorbidity: a systematic review of the literature. Ageing Res Rev. 2011;10(4):430-9.

3. Barat I, Andreasen F, Damsgaard EMS. The consumption of drugs by 75 -year-old individuals living in their own homes. Eur $\mathbf{J}$ Clin Pharmacol. 2000;56(6-7):501-9.

4. Straand J, Fetveit A, Rognstad S, Gjelstad S, Brekke M, Dalen I. A cluster-randomized educational intervention to reduce inappropriate prescription patterns for elderly patients in general practice-The Prescription Peer Academic Detailing (Rx-PAD) study [NCT00281450]. BMC Health Serv Res. 2006;11:6.

5. Ziere G, Dieleman JP, Hofman A, Pols HAP, Van der Cammen TJM, Stricker BHC. Polypharmacyand falls in the middle age and elderly population. Br J Clin Pharmacol. 2006;61(2):218-23.

6. Ziere G, Dieleman JP, Van der Cammen TJM, Hofman A, Pols HAP, Stricker BHC. Selective serotonin reuptake inhibiting antidepressants are associated with an increased risk of nonvertebral fractures. J Clin Psychopharmacol. 2008;28(4):411-7.

7. Stewart RB, Cooper JW. Polypharmacy in the aged-practical solutions. Drugs Aging. 1994;4(6):449-61.

8. Hemminki E, Heikkila J. Elderly peoples' compliance with prescriptions, and quality of medication. Scand J Soc Med. 1975;3 (2):87-92.
9. Bjerrum L, Sogaard J, Hallas J, Kragstrup J. Polypharmacy in general practice: differences between practitioners. Br J Gen Pract. 1999;49(440):195-8.

10. Viktil KK, Blix HS, Moger TA, Reikvam A. Polypharmacy as commonly defined is an indicator of limited value in the assessment of drug-related problems. Br J Clin Pharmacol. 2007;63:187-95.

11. Nyborg G, Straand J, Brekke M. Inappropriate prescribing for the elderly, a modern epidemic? Eur J Clin Pharmacol. 2012;15:1-10.

12. Green JL, Hawley JN, Rask KJ. Is the number of prescribing physicians an independent risk factor for adverse drug events in an elderly outpatient population? Am J Geriatr Pharmacother. 2007;5(1):31-9.

13. Hovstadius B. Petersson G+. Factors leading to excessive polypharmacy. Clin Geriatr Med. 2012;28(2):159-72.

14. Sandvik H. Evaluering av fastlegeordningen 2001-2005 [Summary in English]. Research Council of Norway; 2005.

15. Kann IC, Lundqvist $\mathrm{C}$, Lurå $\mathrm{H}$. Prescription of addictive and non-addictive drugs to home-dwelling elderly. Drugs Aging. 2014;31(6):453-9.

16. WHO Collaborating Centre for Drug Statistics Methodology. Department of Pharmacoepidemiology at the Norwegian Institute of Public Health; 2010. Available from: http://www.whocc.no/ atc_ddd_methodology/who_collaborating_centre/.

17. Patterson SM, Hughes C, Kerse N, Cardwell CR, Bradley MC. Interventions to improve the appropriate use of polypharmacy for older people. Cochrane Database Syst Rev. 2012;5:CD008165. 